\title{
Actin expression in trypanosomatids (Euglenozoa: Kinetoplastea)
}

\author{
Ligia Cristina Kalb Souza, Rosana Elisa Gonçalves Gonçalves Pinho, \\ Carla Vanessa de Paula Lima, Stênio Perdigão Fragoso, Maurilio José Soares/ ${ }^{+}$
}

Instituto Carlos Chagas-Fiocruz, Curitiba, PR, Brasil

\begin{abstract}
Heteroxenic and monoxenic trypanosomatids were screened for the presence of actin using a mouse polyclonal antibody produced against the entire sequence of the Trypanosoma cruzi actin gene, encoding a $41.9 \mathrm{kDa}$ protein. Western blot analysis showed that this antibody reacted with a polypeptide of approximately $42 \mathrm{kDa}$ in the whole-cell lysates of parasites targeting mammals (T. cruzi, Trypanosoma brucei and Leishmania major), insects (Angomonas deanei, Crithidia fasciculata, Herpetomonas samuelpessoai and Strigomonas culicis) and plants (Phytomonas serpens). A single polypeptide of approximately $42 \mathrm{kDa}$ was detected in the whole-cell lysates of $\mathrm{T}$. cruzi cultured epimastigotes, metacyclic trypomastigotes and amastigotes at similar protein expression levels. Confocal microscopy showed that actin was expressed throughout the cytoplasm of all the tested trypanosomatids. These data demonstrate that actin expression is widespread in trypanosomatids.
\end{abstract}

Key words: actin - confocal microscopy - trypanosomatids - Trypanosoma cruzi

Actin is a conserved filamentous protein that plays a major role in diverse eukaryotic cell processes, including cytokinesis and motility. These processes depend on the interactions of actin monomers and filaments with numerous other proteins and are driven by a dynamic remodelling of the actin cytoskeleton, including the assembly of G-actin (a monomeric form) into F-actin (a filamentous form) and the reverse disassembly (Pollard \& Cooper 2009, Lee \& Dominguez 2010). Furthermore, there is increasing evidence, from studies involving a number of cell types, demonstrating the importance of actin during endocytosis (Robertson et al. 2009, Galleta et al. 2010).

Although actin is abundant and well characterised in higher eukaryotic cells, until recently, very little information existed on its expression and distribution in protozoa of the Trypanosomatidae family (Kohl \& Gull 1998). Actin filaments have been only poorly visualised in trypanosomatids through transmission electron microscopy (Sahasrabuddhe et al. 2004). However, their presence has been demonstrated by immunofluorescence in the cytoplasm of the pathogenic species Trypanosoma brucei (García-Salcedo et al. 2004), Trypanosoma cruzi (de Souza et al. 1983, Mortara 1989, Melo et al. 2008, Cevallos et al. 2010) and Leishmania major (Sahasrabuddhe et al. 2004). Interestingly, the immunogenic and filament-forming properties of Leishmania actin differ significantly from those of actin in other eukaryotes, particularly plasmodia, yeast and mammalian actins (Sahasrabuddhe et al. 2004).

doi: 10.1590/0074-0276108052013015

Financial support: CNPq, FIOCRUZ

MJS and SPF receive research fellowships from CNPq.

+ Corresponding author: maurilio@tecpar.br

Received 12 December 2012

Accepted 26 February 2013
The first studies describing the sub-cellular localisation of actin in trypanosomatids were performed using heterologous antibodies against actin from other organisms. Antibodies raised against rabbit actin detected a 43 $\mathrm{kDa}$ protein in T. cruzi and Leishmania mexicana (Mortara 1989) and antibodies raised against Entamoeba histolytica actin showed a positive reaction in the flagellum of T. cruzi (de Souza et al. 1983). More recently, the subcellular localisation of $T$. cruzi actin was evaluated using a homologous antibody and an immunofluorescence analysis of the different developmental forms of this parasite (epimastigotes, bloodstream trypomastigotes and amastigotes). This assay revealed many rounded and punctuated structures, resembling patches, distributed throughout the cytoplasm, which had no association with subpellicular microtubules or with the nuclear or kinetoplast DNA (Melo et al. 2008). Actin-like filaments were also demonstrated in L. major using a homologous antibody and actin was present in the promastigote form as granules, as short filaments or bundles and as patches (Sahasrabuddhe et al. 2004).

Here, we have screened pathogenic and non-pathogenic species of trypanosomatids for the presence of actin by western blotting using a homologous antibody coded with the entire sequence of the $T$. cruzi actin gene (TcActin). Furthermore, we have analysed the expression of this protein in different developmental forms of $T$. cruzi (cultured epimastigotes, in vitro-derived trypomastigotes and cell culture-derived amastigotes) and studied its subcellular localisation by confocal microscopy.

\section{MATERIALS AND METHODS}

Reagents - Bovine serum albumin (BSA) was purchased from Sigma (Sigma Chemical Co, St. Louis, MO, USA). Alexa Fluor 488-conjugated rabbit anti-mouse immunoglobulin $\mathrm{G}(\mathrm{IgG})$ was purchased from Molecular Probes (Carlsbad, CA, USA). Horseradish peroxidase (HRP)-conjugated rabbit anti-mouse IgG was purchased from Santa Cruz Biotechnology (Santa Cruz, CA, USA). 
Molecular weight markers (Benchmark Protein Ladder, 10-220 kDa) and the fluorescent dye Hoechst 33342 trihydrochloride were purchased from Invitrogen (Eugene, OR, USA). A polyclonal mouse antibody against $T$. cruzi glyceraldehyde 3-phosphate dehydrogenase (GAPDH) was kindly provided by $\mathrm{F}$ Morini [Carlos Chagas Institute, Oswaldo Cruz Foundation (Fiocruz), state of Paraná, Brazil].

Parasites - T. cruzi clone Dm28c (Contreras et al. 1988) epimastigote forms were grown at $28^{\circ} \mathrm{C}$ in liver infusion tryptose (LIT) medium (Camargo 1964) supplemented with $10 \%$ foetal bovine serum (FBS), with weekly passages. Epimastigotes were differentiated in vitro into metacyclic trypomastigotes in triatomine artificial urine (TAU)3AAG medium (190 mM NaCl, 17 $\mathrm{mM} \mathrm{KCl}, 2 \mathrm{mM} \mathrm{MgCl}, 2 \mathrm{mM} \mathrm{CaCl}$ and $8 \mathrm{mM}$ phosphate buffered saline (PBS) at $\mathrm{pH} 6.0$ supplemented with $10 \mathrm{mM}$ L-proline, $50 \mathrm{mM}$ L-sodium glutamate, $2 \mathrm{mM}$ L-sodium aspartate and $10 \mathrm{mM} \mathrm{D}$-glucose) (Contreras et al. 1985). Briefly, five-day-old cultured epimastigotes were harvested by centrifugation for $10 \mathrm{~min}$ at $8,500 \mathrm{~g}$. The parasites were then incubated at a density of $5 \mathrm{x}$ $10^{8}$ cells $/ \mathrm{mL}$ for $2 \mathrm{~h}$ at $28^{\circ} \mathrm{C}$ in TAU medium. Cells (at a 1:100 dilution) were further incubated for $96 \mathrm{~h}$ at $28^{\circ} \mathrm{C}$ in culture flasks that contained no more than $1 \mathrm{~cm}$ deep of TAU3AAG medium. The number of living epimastigotes and metacyclic trypomastigotes was then determined by cell counting via a Neubauer chamber after 24 $\mathrm{h}, 48 \mathrm{~h}$ and $72 \mathrm{~h}$. The metacyclic trypomastigotes were purified from the $72 \mathrm{~h}$-old cultures by DEAE-51 cellulose chromatography (Contreras et al. 1985).

To obtain amastigote forms, metacyclic trypomastigotes derived in vitro were used to infect Vero cells (ATCC CRL-1586), which were then cultivated in Dulbecco's Modified Eagle's medium (Sigma) supplemented with $5 \% \mathrm{FBS}$ and incubated in a humidified atmosphere with $5 \% \mathrm{CO}_{2}$ at $37^{\circ} \mathrm{C}$. After 10 days of infection, the amastigotes released into the supernatant were harvested by centrifugation at $1,000 \mathrm{~g}$ for $5 \mathrm{~min}$.

L. major promastigotes were grown at $28^{\circ} \mathrm{C}$ in Schneider's medium (Sigma) supplemented with 10\% FBS and $10 \mathrm{mg} / \mathrm{L}$ haemin. Procyclic trypomastigote forms of T. brucei were cultivated at $28^{\circ} \mathrm{C}$ in SDM79 medium (Invitrogen) supplemented with $10 \% \mathrm{FBS}$ and $10 \mathrm{mg} / \mathrm{L}$ haemin (Brun \& Schonenberger 1979). Strigomonas culicis (formerly Blastocrithidia culicis) (Teixeira et al. 2011), Angomonas deanei (formerly Crithidia deanei) (Teixeira et al. 2011) with its bacterial endosymbiont, Crithidia fasciculata and Herpetomonas samuelpessoai were grown at $28^{\circ} \mathrm{C}$ in LIT medium supplemented with $10 \%$ FBS with daily passages. Phytomonas serpens was grown at $28^{\circ} \mathrm{C}$ in GYPMY medium (Itow-Jankevicius et al. 1993) with passages every three days.

Cloning and expression of TcActin - The complete coding sequence of TcActin (Dm28c clone) was amplified using synthetic primers that were based on the T. cruzi CL Brener sequence (accession Tc00.1047053510571.30) available from the T. cruzi genome database (genedb. org). The putative open reading frame of 1,131 bp, encoding a protein of $41.9 \mathrm{kDa}$, was amplified by poly- merase chain reaction (PCR) using forward primer 5 'CGGAATTCATGTCTGACGAAGAACAGTCCGCTATT3', reverse primer 5'ATTCGTCGACTCATTGT TGTGCACAATGCTTGGGCCTGCCTCGTC3' and the genomic DNA as a template. The PCR products, flanked by EcoRI and Sall sites, were inserted into a vector (pGEX-4 $\mathrm{T}_{1}$, Invitrogen) containing glutathioneS-transferase (GST). Positive clones were purified with a QIAprep Spin Miniprep kit (QIAGEN, Valencia, CA, USA) and sequencing was performed at Macrogen Inc (Seoul, Korea). The correct sequence was confirmed by the BLAST algorithm. The amplified fragments inserted into the pGEX-4T, vector (Invitrogen) were used to transform Escherichia coli strain Top 10. Production of the recombinant protein was induced by adding $0.3 \mathrm{mM}$ isopropyl- $\beta-D-1$-thiogalactopyranoside. Inclusion bodies containing the recombinant protein were isolated and the recombinant protein was purified by electro-elution.

Polyclonal antiserum - A polyclonal antiserum against the expressed recombinant TcActin was produced in albino Swiss mice. Approximately $50 \mu \mathrm{g}$ of purified, GST-tagged TcActin was mixed 1:1 with complete Freund's adjuvant and inoculated intraperitoneally in mice. After 15 days, the animals received three consecutive boosts at two-week intervals, each containing an additional $20 \mu \mathrm{g}$ of the antigen and $77 \mu \mathrm{L}$ of Alu-Gel (Serva, Heidelberg, Germany) as adjuvant. The antiserum was harvested five days after the last booster injection. This study was carried out in strict accordance with the recommendations in the guide for animal use of the Fiocruz and the protocol was approved by the Committee on Animal Experimentation (P-0434/07).

Western blot analysis - For immunoblotting analysis, all of the parasites' total protein extracts were prepared by resuspending the PBS-washed parasites $\left(10^{6}\right.$ cells/ $\mu \mathrm{L})$ in sodium dodecyl sulphate polyacrylamide gel electrophoresis (SDS-PAGE) sample buffer to lyse the cells $\left(10^{7}\right.$ cells/lane). The proteins were fractionated in $13 \%$ polyacrylamide gels by SDS-PAGE and transferred onto nitrocellulose membranes (Hybond C, Amersham Biosciences, England), according to standard protocols (Sambrook et al. 1989). Following the transfer of the proteins to nitrocellulose, the membranes were blocked with 5\% non-fat milk and $0.05 \%$ Tween- 20 in PBS. The membranes were then incubated for $2 \mathrm{~h}$ with a blocking buffer containing the polyclonal antiserum raised against recombinant protein TcActin (1:200 dilution). After three washings with $0.05 \%$ Tween-20 in PBS, the nitrocellulose was incubated for $1 \mathrm{~h}$ with HRP-conjugated rabbit anti-mouse $\operatorname{IgG}$ (1:10,000 dilution). The membrane was again washed three times with $0.05 \%$ Tween- 20 in PBS and the reactive bands were visualised using the BCIPNBT solution, as described by the manufacturer.

Furthermore, $15 \mu \mathrm{g}$ of the total proteins obtained from the T. cruzi cultured epimastigotes, metacyclic trypomastigotes and cell culture-derived amastigotes, as well as those from the Vero cells, were resolved on SDS-PAGE (13\%) gels. Following the transfer of the proteins to nitrocellulose, the membrane was incubated for $2 \mathrm{~h}$ with a blocking buffer containing both the polyclonal 
antiserum raised against recombinant protein TcActin (1:200 dilution) and a polyclonal antiserum against $T$. cruzi GAPDH. After three washings with $0.05 \%$ Tween20 in PBS, the membranes were incubated as described above. The relative amount of actin and GAPDH (1:250 dilution) expressed by each developmental stage was estimated using ImageJ software (National Institutes of Health, Bethesda, MD, USA).

Immunofluorescence assays - All of the parasites (including T. cruzi epimastigotes, amastigotes and metacyclic trypomastigotes) were washed and resuspended at a density of $10^{7}$ cells $/ \mathrm{mL}$ in PBS. The cells were fixed with $4 \%$ paraformaldehyde for $15 \mathrm{~min}$ at room temperature (RT), washed twice in PBS and then adhered to $0.1 \%$ poly-L-lysine-coated coverslips, which were themselves incubated for $20 \mathrm{~min}$ at RT. The cells were made permeable by 2 min of incubation with $0.1 \%$ Triton X-100 in PBS; next, they were washed with PBS, blocked by incubation overnight with $1 \%$ BSA in PBS and incubated for $1 \mathrm{~h}$ with the polyclonal antiserum against TcActin (1:150 dilution). After another washing, the samples were incubated with Alexa Fluor 488-conjugated rabbit anti-mouse $\mathrm{IgG}$ at a 1:800 dilution. The nuclear and kinetoplast DNA were stained by incubation with Hoechst 33342. After extensive washing, the coverslips were mounted onto glass microscope slides using N-propyl-gallate for anti-fading. The samples were examined using a Leica SP5 confocal laser microscope (Leica Microsystems, Mannheim, Germany) and the images were processed for better contrast using Adobe Photoshop CS3 software.

\section{RESULTS}

A polypeptide of approximately $41.9 \mathrm{kDa}$, encoded by the entire sequence of the TcActin, was used to obtain a polyclonal TcActin antibody prepared in mice. When used in western blotting to probe for the presence of actin in trypanosomatids, this antibody reacted with a peptide of approximately $42 \mathrm{kDa}$ in the whole-cell lysates of multiple species (Fig. 1A), including parasites targeting mammals (T. cruzi epimastigotes, T. brucei procyclic forms and L. major promastigotes), insects (A. deanei, C. fasciculata and S. culicis) and plants (P. serpens). Interestingly, two close weak bands near that weight were recognised in the $P$. serpens blot.

The expression of actin was also analysed by western blotting in different life stages of $T$. cruzi, as well as in Vero cells, using the TcActin antibody (Fig. 1B). This procedure identified a single peptide of approximately $42 \mathrm{kDa}$ (Fig. 1B, upper bands) in the whole-cell lysates of all tested T. cruzi stages (culture epimastigotes, metacyclic trypomastigotes and cell-derived amastigotes). A similar actin expression level was observed in all of the developmental forms, as confirmed by band densitometry analysis conducted using ImageJ software (Fig. 1C). The GAPDH banding pattern (Fig. 1B, lower bands) was used as a loading control.

Confocal microscopy and the TcActin antibody were used to visualise the cellular distribution of actin in different trypanosomatids, both monoxenic (A. dea$n e i$ with its bacterial endosymbiont, $C$. fasciculata, $H$. samuelpessoai and S. culicis) and heteroxenic (P. serpens). In the tested parasites, this antibody recognised the protein in all of the cells in the population (data not shown). The positive reaction was homogeneously dispersed throughout the cytoplasm of all the cells (Fig. 2). No reaction was observed in the flagellum. In A. deanei, the labelling was more intense at the bacterial endosymbionts, which are located at the posterior cell region.

In T. cruzi, punctate labelling for TcActin was found throughout the cytoplasm of epimastigotes, metacyclic trypomastigotes and amastigotes. No reaction was observed in microtubule-containing structures such as the flagellum and the plasma membrane (Fig. 3). In the trypomastigotes, the positive granules were arranged in linear arrays (Fig. $3 \mathrm{H})$. In the epimastigotes and amastigotes, positive reactions were occasionally found in elongated structures that may represent the cytostome/ cytopharynx (Fig. 3B).

\section{DISCUSSION}

In the present study, a mouse TcActin polyclonal antibody was cross-reacted with different trypanosomatids, including some monoxenic species found in insects $(A$. deanei, C. fasciculata, H. samuelpessoai and S. culicis) as well as a plant species (P. serpens). Both the presence of actin genes and the expression of the encoded protein have previously been demonstrated in some pathogenic heteroxenic trypanosomatids, such as T. brucei (GarcíaSalcedo et al. 2004), T. evansi (Li et al. 2009), T. cruzi (de Souza et al. 1983, Mortara 1989, Melo et al. 2008), T. rangeli (Toaldo et al. 2001) and L. major (Sahasrabuddhe et al. 2004). The cross-reaction of our TcActin polyclonal antibody with $T$. brucei procyclic forms and L. major promastigotes, both of which are parasites known to ex-
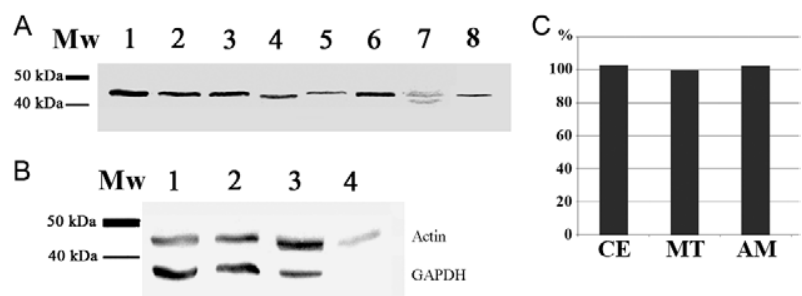

Fig. 1: western blot using the Trypanosoma cruzi actin gene (TcActin) antibody. A: different trypanosomatids were probed with detection of a single band of about $42 \mathrm{kDa}$, except for Phytomonas serpens (Lane 7) where two close faint bands could be observed. Lanes 1-8 contained $10^{7}$ cells (1: T. cruzi; 2: Trypanosoma brucei; 3: Leishmania major; 4: Strigomonas culicis; 5: Crithidia fasciculata; 6: Angomonas deanei with symbiont; 7: Phytomonas serpens; 8: Herpetomonas samuelpessoai); B: different T. cruzi stages probed against the anti-TcActin antibody (upper bands) and an anti-glyceraldehyde 3-phosphate dehydrogenase antibody (lower bands) [1: culture epimastigote (CE) in liver infusion tryptose (LIT) medium; 2: metacyclic trypomastigote (MT); 3: amastigote (AM); 4: Vero cells (actin control); 1-4 contained 15 $\mu \mathrm{g}$ protein/lane]; C: actin expression level as evaluated by integrated density of protein bands (using GAPDH band as normaliser) analysed by the ImageJ software $(n=3)$. To evaluate the actin expression in $\mathrm{AM}$, the Vero cell band signal was subtracted from the AM band signal; MW: molecular weight markers. 
press actin (García-Salcedo et al. 2004, Sahasrabuddhe et al. 2004), validated our polyclonal antiserum. Taken together, these data indicate that actin is present and conserved throughout the Trypanosomatidae family.

In a previous work, an antiserum raised against the entire actin from $T$. cruzi recognised a single band in one-dimensional electrophoresis. However, five actin isoforms were identified by this antiserum in two-dimensional electrophoresis (Cevallos et al. 2010). Therefore, it seems plausible that our polyclonal antibody (produced against the entire actin sequence) also recognised multiple actin isoforms within a single band generated by one-dimensional electrophoresis. The presence of two reacting bands in the $P$. serpens lysate suggests that this parasite possesses actin isoforms of different sizes.

In a previous study, an anti-Leishmania actin antibody was cross-reacted with cell lysates of various Leishmania species as well as of T. cruzi. However, that antibody failed to recognise the actin bands in the wholecell lysates of Plasmodium falciparum, Saccharomyces cerevisiae, BHK21 cells and human erythrocytes, in-

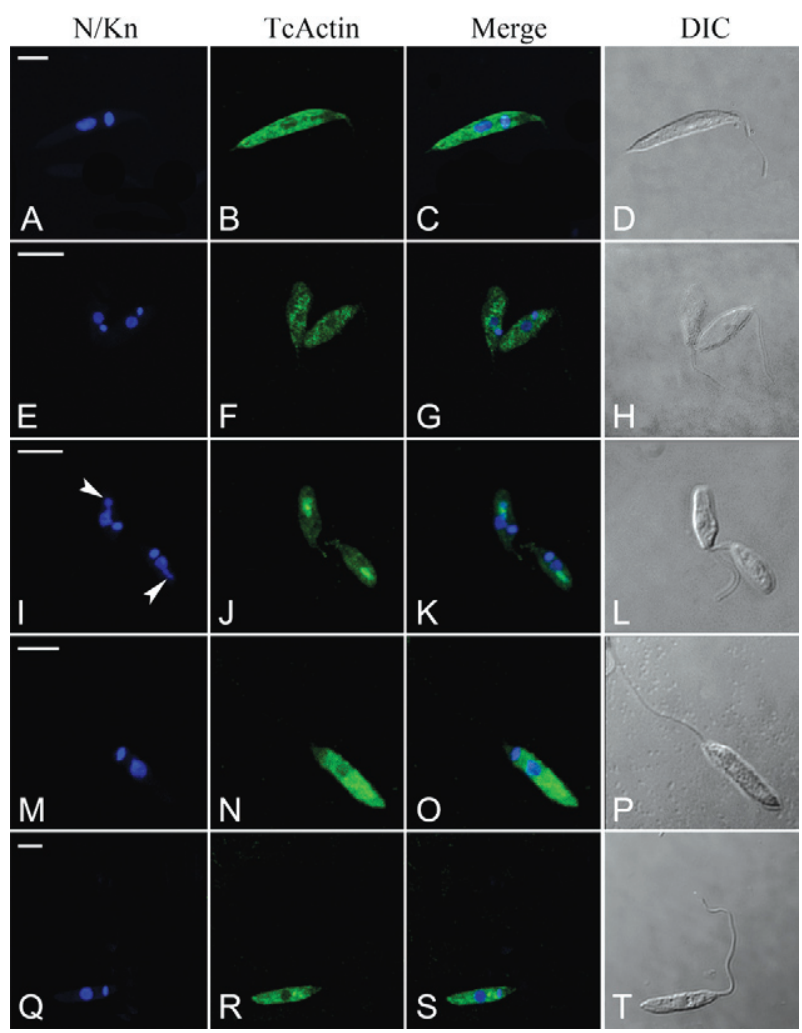

Fig. 2: actin localisation in different trypanosomatids by confocal microscopy by using the Trypanosoma cruzi actin gene (TcActin) antibody (actin detection with TcActin mouse polyclonal antibody plus Alexa Fluor 488-conjugated rabbit anti-mouse immunoglobulin G; note the staining throughout the cell body). A-D: Strigomonas culicis; DIC: differential interference contrast images of the parasite body; E-H: Crithidia fasciculata; I-L: Angomonas deanei with symbiont; Merge: merged Hoechst and actin images; M-P: Phytomonas serpens; N/Kn: staining of nuclear $(\mathrm{N})$ and kinetoplast $(\mathrm{Kn})$ DNA with Hoechst 33342; Q-T: Herpetomonas samuelpessoai. Arrowheads in I indicate staining of the bacterial endosymbiont. Bar $=5 \mu \mathrm{m}$. dicating that the actin of trypanosomatids is divergent from that of other eukaryotic cells (Sahasrabuddhe et al. 2004). Accordingly, a rabbit anti-human $\alpha$-actin polyclonal antibody readily recognised the actin band in wholecell lysates of P. falciparum, S. cerevisiae, BHK21 cells and human erythrocytes, but did not cross-react with Leishmania or Trypanosoma actin (Sahasrabuddhe et al. 2004). A polyclonal antibody against a conserved Cterminal peptide of rabbit actin only poorly recognised $T$. cruzi actin. However, an antibody against T. cruzi actin was not species-specific, as proteins of a similar size were also detected in lysates from L. mexicana procyclics, Toxoplasma gondii sporozoites and Trichomonas vaginalis trophozoites (Cevallos et al. 2010).

The homology levels of $T$. cruzi actin compared with the eukaryotic actins of L. major, T. brucei, P. falciparum, Dictyostelium discoideum and Homo sapiens are $95 \%, 99 \%, 71 \%, 69 \%$ and $86 \%$, respectively (Melo et al. 2008). Thus, the properties of trypanosomatid actin differ from those of the yeast, Plasmodium and human actins. The major differences were confined to the amino acids located on the surfaces of the yeast and mammalian actins, including amino acid residues 42-46, which constitute the major self-association sites for actin filament formation (Sahasrabuddhe et al. 2004). F-actin has not yet been clearly identified in trypanosomatids, which might be due to filament instability, deficient assembly or accentuated severing, events that are not yet understood (Melo et al. 2008).

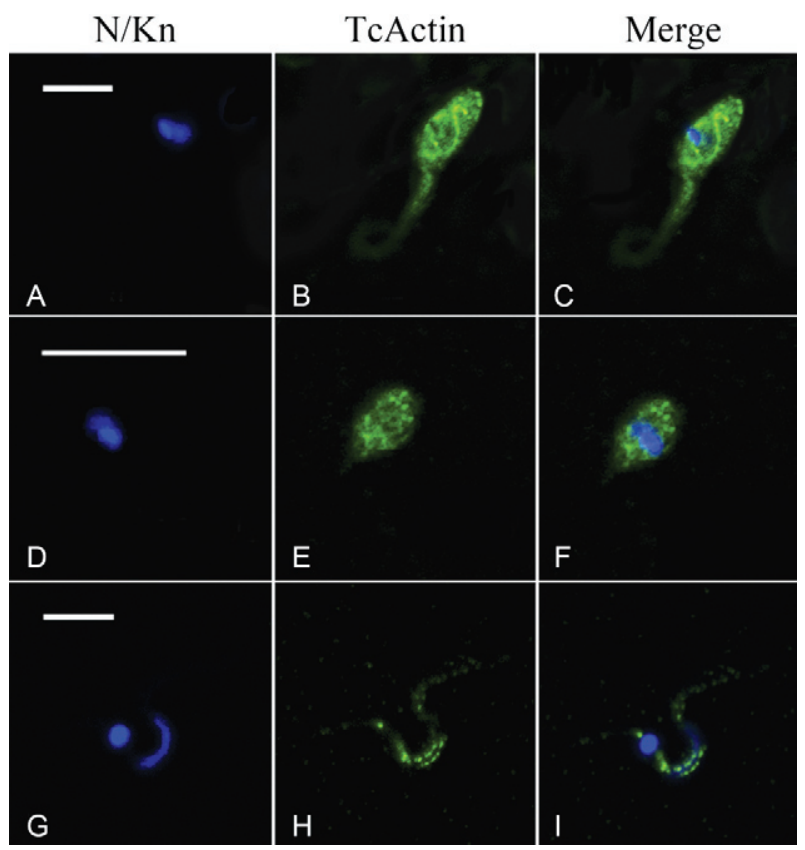

Fig. 3: actin localisation in Trypanosoma cruzi by using the T. cruzi actin gene (TcActin) antibody (actin detection with TcActin mouse polyclonal antibody plus Alexa Fluor 488-conjugated rabbit antimouse immunoglobulin G). A-C: epimastigote form (note the positive labelling at an elongated structure that may represent the cytopharynx); D-F: amastigote form; G-I: trypomastigote form; N/Kn: staining of nuclear (N) and kinetoplast (Kn) DNA with Hoechst 33342; Merge: merged Hoechst and actin images. Bar $=5 \mu \mathrm{m}$. 
With confocal microscopy, we found positive reactions for actin dispersed throughout the cytoplasm of all the tested trypanosomatids. This cytoplasmic pattern has also been observed in Leishmania sp. (Sahasrabuddhe et al. 2004), T. cruzi (de Souza et al. 1983, Mortara 1989, Melo et al. 2008, Cevallos et al. 2010) and T. brucei (García-Salcedo et al. 2004). A previous study described the subcellular localisation of actin in T. cruzi by using an antibody produced against an actin fragment containing the conserved N-terminal region (SKKLFVGDEAQAKR), which is present in all three T. cruzi actin alleles available from GenBank (Melo et al. 2008). This experiment resulted in actin labelling throughout the parasite cell body. In the present work, we have confirmed the cellular localisation of actin in $T$. cruzi epimastigotes, trypomastigotes and amastigotes by using an antibody produced against the entire actin coding region. Furthermore, our data indicate that actin is expressed at similar levels in these developmental stages. It has previously been shown that actin is equally expressed in different evolutive forms of T. brucei (GarcíaSalcedo et al. 2004). Thus, actin can act as an optional normaliser for protein expression studies investigating the $T$. cruzi differentiation process.

This is the first study to describe actin localisation in T. cruzi metacyclic trypomastigotes, as previous works have analysed actin distribution only in cell-derived trypomastigotes (Cevallos et al. 2010) or bloodstream trypomastigotes (Melo et al. 2008). Although similar in their distribution of cellular organelles, these forms constitute different stages of the parasite. Thus, the linear array of actin granules observed in metacyclic trypomastigotes is interesting and may represent a distinct actin arrangement.

An increased signal was detected near the bacterial endosymbionts located at the posterior cell end of $A$. dea$n e i$, suggesting that some type of actin-based cytoskeleton might be related to the symbionts' positioning in this region. It has been previously speculated that such an actin-based cytoskeleton might also be related to anchoring RNA granule components in $T$. cruzi, as granules that remain motionless were associated with actin filaments, whereas those that moved in the cytoplasm remained associated with microtubules (Holetz et al. 2010).

Some studies of trypanosomes showed that actin participates in endocytosis and intracellular transport in these organisms (García-Salcedo et al. 2004). Thus, a repression of actin expression by iRNA in the bloodstream forms of the African trypanosome T. brucei resulted in the cessation of growth, loss of the endocytic activity and termination of vesicular traffic from the flagellar pocket membrane (García-Salcedo et al. 2004). Whereas in procyclic forms, actin was uniformly distributed in the cytoplasm, in bloodstream forms, actin was primarily located at the posterior end of the cell, clearly concentrated between the nucleus and the kinetoplast, a site of highly active endocytic activity (García-Salcedo et al. 2004). A proteomic analysis of the American trypanosome T. cru$z i$ indicated the presence of actin in the reservosome, an end organelle of the endocytic pathway (Sant'Anna et al. 2009). Accordingly, thus far, the only demonstrated func- tional role of actin in T. cruzi has been related to endocytosis (Bogitsh et al. 1995, García-Salcedo et al. 2004). These data reinforce evidence from other eukaryotic cells indicating the importance of an actin cytoskeleton during endocytosis (Robertson et al. 2009, Galleta et al. 2010). The possible localisation of actin at the cytostome/ cytopharynx of $T$. cruzi epimastigotes would imply the presence of a contractile cytoskeleton at this region. Further experiments are needed to confirm this hypothesis.

\section{ACKNOWLEDGEMENTS}

To Nilson Fidêncio, Vanessa Stella and Crisciele Kuligovski, for technical assistance.

\section{REFERENCES}

Bogitsh BJ, Ribeiro-Rodrigues R, Carter CE 1995. In vitro effects of mannan and cytochalasin $\mathrm{B}$ on the uptake of horseradish peroxidase and $\left[{ }^{14} \mathrm{C}\right]$ sucrose by Trypanosoma cruzi epimastigotes. J Parasitol 81: 144-148.

Brun R, Schonenberger M 1979. Cultivation and in vitro cloning of procyclic culture forms of Trypanosoma brucei in a semi-defined medium. Acta Trop 36: 289-292.

Camargo EP 1964. Growth and differentiation in Trypanosoma cruzi. I. Origin of metacyclic trypanosomes in liquid media. Rev Inst Med Trop Sao Paulo 12: 93-100.

Cevallos AM, Segura-Kato YX, Merchant-Larios H, Manning-Cela R, Alberto Hernández-Osorio L, Márquez-Dueñas C, Ambrosio JR, Reynoso-Ducoing O, Hernández R 2010. Trypanosoma cruzi: multiple actin isovariants are observed along different developmental stages. Exp Parasitol 127: 249-259.

Contreras VT, Araújo-Jorge TC, Bonaldo MC, Thomaz N, Barbosa HS, Meirelles MNSL, Goldenberg S 1988. Biological aspects of the DM 28c clone of Trypanosoma cruzi after metacyclogenesis in chemically defined media. Mem Inst Oswaldo Cruz 83: 123-133.

Contreras VT, Salles JM, Thomas N, Morel CM, Goldenberg S 1985. In vitro differentiation of Trypanosoma cruzi under chemically defined conditions. Mol Biochem Parasitol 16: 315-327.

de Souza W, Meza I, Martinez-Palomo A, Sabanero M, Souto-Padrón T, Meirelles MN 1983. Trypanosoma cruzi: distribution of fluorescently labeled tubulin and actin in epimastigotes. J Parasitol 69: 138-142.

Galletta BJ, Mooren OL, Cooper JA 2010. Actin dynamics and endocytosis in yeast and mammals. Curr Opin Biotechnol 21: 604-610.

García-Salcedo JA, Pérez-Morga D, Gijón P, Dilbeck V, Pays E, Nolan DP 2004. A differential role for actin during the life cycle of Trypanosoma brucei. EMBO J 23: 780-789.

Holetz FB, Alves LR, Probst CM, Dallagiovanna B, Marchini FK, Manque P, Buck G, Krieger MA, Correa A, Goldenberg S 2010. Protein and mRNA content of TcDHH1 containing mRNPs in Trypanosoma cruzi. FEBS J 277: 3415-3426.

Itow-Jankevicius S, Almeida ML, Jankevicius JV, Cavazzana Jr M, Attias M, de Souza W 1993. Axenic cultivation of trypanosomatids found in corn (Zea mays) and in phytophagous hemipterans (Leptoglossus zonatus - Coreidae) and their experimental transmission. J Eukaryot Microbiol 40: 576-581.

Kohl L, Gull K 1998. Molecular architecture of the trypanosome cytoskeleton. Mol Biochem Parasitol 93: 1-9.

Lee SH, Dominguez R 2010. Regulation of actin cytoskeleton dynamics in cells. Mol Cells 29: 311-325.

Li SQ, Yang WB, Ma LJ, Xi SM, Chen QL, Song XW, Kang J, Yang LZ 2009. Immunization with recombinant actin from Trypanosoma 
evansi induces protective immunity against $T$. evansi, T. equiperdum and T. b. brucei infection. Parasitol Res 104: 429-435.

Melo LDB, Sant'anna C, Reis SA, Lourenço D, de Souza W, Lopes UG, Cunha-e-Silva NL 2008. Evolutionary conservation of actinbinding proteins in Trypanosoma cruzi and unusual subcellular localization of the actin homologue. Parasitology 135: 955-965.

Mortara RA 1989. Studies on trypanosomatid actin. I. Immunochemical and biochemical identification. J Protozool 36: 8-13.

Pollard TD, Cooper JA 2009. Actin, a central player in cell shape and movement. Science 326: 1208-1212.

Robertson AS, Smythe E, Ayscough KR 2009. Functions of actin in endocytosis. Cell Mol Life Sci 66: 2049-2065.

Sahasrabuddhe AA, Bajpai VK, Gupta CM 2004. A novel form of actin in Leishmania: molecular characterization, subcellular localization and association with subpellicular microtubules. $\mathrm{Mol}$ Biochem Parasitol 134: 105-114.
Sambrook J, Fritsch EF, Maniatis T 1989. Detection and analysis of proteins expressed from cloned genes. In J Sambrook, EF Fritsch, T Maniatis, Molecular cloning: a laboratory manual, 2nd ed., Vol. 3, Cold Spring Harbor Laboratory Press, Woodbury, p. 18.60-18.74.

Sant'Anna C, Nakayasu ES, Pereira MG, Lourenço D, de Souza W, Almeida IC, Cunha-e-Silva NL 2009. Subcellular proteomics of Trypanosoma cruzi reservosomes. Proteomics 7: 1782-1794.

Teixeira MM, Borghesan TC, Ferreira RC, Santos MA, Takata CS, Campaner M, Nunes VL, Milder RV, de Souza W, Camargo EP 2011. Phylogenetic validation of the genera Angomonas and Strigomonas of trypanosomatids harboring bacterial endosymbionts with the description of new species of trypanosomatids and of proteobacterial symbionts. Protist 162: 503-524.

Toaldo CB, Steindel M, Sousa MA, Tavares CC 2001. Molecular karyotype and chromosomal localization of genes encoding $\beta$-tubulin, cysteine proteinase, hsp 70 and actin in Trypanosoma rangeli. Mem Inst Oswaldo Cruz 96: 113-121. 